\title{
LA PRÁCTICA PEDAGÓGICA DE UN PROFESOR DE PORTUGUÉS LENGUA EXTRANJERA (PLE) EN CONTEXTO DE INMERSIÓN Y NO- INMERSIÓN
}

\author{
Nildicéia Aparecida ROCHA ${ }^{1}$ \\ Rosangela Sanches da Silveira GILENO ${ }^{2}$
}

Resumen: El objetivo de este trabajo es presentar una reflexión sobre las especificidades de enseñar Portugués Lengua Extranjera (PLE) en contexto de inmersión y fuera de él, a partir de observaciones realizadas en dos momentos: inicialmente, se describirá la práctica de un profesor de PLE en situación de inmersión en una universidad en el interior del estado de São Paulo, Brasil; posteriormente, la práctica del mismo profesor en un curso de PLE fuera del contexto de inmersión, en una universidad en España, en una capital de provincia. En este sentido, será analizada la práctica docente de un profesor de PLE en situación de inmersión y fuera de ella, partiendo del tratamiento didáctico-pedagógico sobre la relación indisoluble entre lengua y cultura, bajo el enfoque comunicativo intercultural, y con el objetivo de desarrollar en el aprendiz una competencia comunicativa intercultural. Se trata de una investigación cualitativa, un estudio de caso, centrada en la práctica de un mismo profesor (sujeto de la investigación) en dos contextos. Los resultados apuntan que la práctica del profesor en contexto de no-inmersión tuvo que ser resignificada para posibilitar el aprendizaje más significativo de PLE en tal contexto. De hecho, la docente redefinió su práctica y tuvo que desconstruir una "creencia" empírica y se abocó hacía la teoría, verificando que el análisis de datos sociales, históricos y culturales deben ser determinantes en la enseñanza PLE fuera de inmersión, principalmente cuando se propone la enseñanza a partir de la interrelación entre lengua y cultura.

Palabras-clave: Práctica pedagógica. Enseñanza de portugués lengua extranjera. Contexto de inmersión. Lengua y cultura.

\section{INTRODUÇÃO}

Este trabajo se propone presentar una reflexión sobre las especificidades de enseñar Portugués Lengua Extranjera (PLE) tanto en contexto de inmersión como fuera del mismo, a partir de las observaciones y análisis realizados en dos momentos: primero se describirá la práctica de un profesor de PLE en situación de inmersión en una universidad en el interior del estado de São Paulo, Brasil; y, en un segundo momento, la práctica del mismo profesor en un curso de PLE fuera del contexto de inmersión, en una

\footnotetext{
${ }^{1}$ Docente. Departamento de Letras Modernas. UNESP - Universidade Júlio de Mesquita Filho. Faculdade de Ciências e Letras. Araraquara - SP - Brasil. 14800-901- nildirocha@ fclar.unesp.br.

${ }^{2}$ Docente. Departamento de Didática. UNESP - Universidade Júlio de Mesquita Filho. Faculdade de Ciências e Letras. Araraquara - SP - Brasil. 14800-901- rosangela@ fclar.unesp.br.
} 
universidad española de una capital de provincia. En este sentido, analizaremos la práctica docente de un profesor de PLE en situación de inmersión y fuera de ella, cuando el tratamiento didáctico-pedagógico parte de la relación indisoluble entre lengua y cultura, bajo el enfoque comunicativo intercultural, con el propósito de desarrollar en el aprendiz una competencia comunicativa intercultural.

De ese modo, este trabajo se propone, inicialmente, promover una discusión teórica y metodológica sobre la enseñanza y el aprendizaje en contextos de inmersión y fuera de él para, posteriormente, presentar el contexto institucional y pedagógico de los cursos especificados previamente. Una vez hecha la descripción de los contextos y el levantamiento de los datos en dichos contextos, se realizará la interpretación analítica de los datos observados y se finalizará con algunas reflexiones que tienden a promover nuevos estudios.

\section{Fundamentación teórica}

La hipótesis de este trabajo consiste en que enseñar una lengua extranjera en situación de inmersión puede presentar una importante ventaja en relación a enseñar fuera de inmersión. Además, consideramos que estar en inmersión o fuera de ella puede ser determinante para la enseñanza, llevando en consideración la interrelación entre cultura y lengua. No obstante, surge la siguiente pregunta: ¿en qué medida la situación de inmersión puede favorecer tal enseñanza o aprendizaje, en la articulación entre lengua y cultura?

La teoría hace hincapié, desde hace algunos años, en la necesidad de enseñarse una lengua a partir de la relación indisociable que existe entre esta y su cultura. Gran parte de los trabajos ya observan las cuestiones relacionadas a la educación intercultural (KRAMSCH, 1993); al enfoque intercultural (MENDES, 2008) y a la competencia comunicativa intercultural (BYRAM, 1997).

Se puede decir que la educación intercultural intenta transformar las barreras culturales en puentes interculturales. Así, un abordaje dialógico, bajo el interculturalismo, puede crear enlaces o vínculos entre la lengua y la cultura, por medio de la exploración entre las fronteras interculturales creadas por la lengua en la construcción de la realidad. En la perspectiva de la enseñanza comunicativa para las lenguas extranjeras, Mendes (2004) presenta el concepto de Enfoque Comunicativo Intercultural (en portugués, Abordagem Comunicativa Intercultural, ACIN) como: 
A força potencial que pretende orientar as ações de professores, alunos e de outros envolvidos no processo de ensino/aprendizagem de uma língua, materna ou estrangeira, o planejamento de cursos, a produção de materiais e a avaliação da aprendizagem, com o objetivo de promover a construção conjunta de significados para um diálogo entre culturas [...] portanto [...] orienta um modo de ser e de agir, de ensinar e de aprender, de produzir planejamentos e materiais culturalmente sensíveis aos sujeitos participantes do processo de aprendizagem, em busca da construção de um diálogo intercultural. (MENDES, 2008, p.60).

Conviene resaltar que lo que Mendes (2008) considera intercultural son las acciones, actitudes y prácticas que hacen que los alumnos se valoren respeto al otro, así como las diferencias y la diversidad cultural, promoviendo la construcción de nuevos significados a través de la interacción entre sus experiencias que vienen de la cooperación e integración de mundos culturales, en ocasiones, diferentes. La autora presenta tres principios fundamentales del enfoque comunicativo intercultural (MENDES, 2008): el primero, se relaciona con la alteridad, o sea, el modo cómo vemos al otro y al mundo que nos rodea; el segundo, dialoga con la identidad, refiriéndose a cómo actuamos en el mundo y cómo dividimos nuestra experiencia; y el tercero es una unión de los dos anteriores, pues expone cómo nos comunicamos con el otro.

Según el enfoque comunicativo intercultural, es necesario desarrollar una competencia comunicativa intercultural (CCI). Para Byram (1997), la competencia intercultural se refiere a la habilidad de los hablantes de comunicarse en sus propias lenguas, con otras lenguas y culturas. La competencia intercultural, sumada a las otras competencias, como la lingüística, la sociolingüística y la discursiva, componen la competencia comunicativa intercultural. Partiendo de tales presupuestos y ubicándonos en el tema central de este estudio, la enseñanza de Portugués Lengua Extranjera con foco en temas culturales y en un enfoque comunicativo intercultural, la cuestión es: ¿estar en contexto de inmersión favorece la enseñanza y el aprendizaje o estar fuera de la situación de inmersión dificulta este proceso?

De acuerdo con los aportes teóricos de Krashen (1985), con relación a la distinción entre adquisición y aprendizaje de lenguas extranjeras, podemos decir que la situación de adquisición favorece el desarrollo de la competencia comunicativa del extranjero. En condiciones ideales, dado que la adquisición es producto de un proceso subconsciente que requiere interacción significativa en la lengua-meta, lo que él denomina de comunicación natural, donde los hablantes están concentrados no en la forma de los enunciados y sí en el acto comunicativo (KRASHEN, 1985). 
En otras palabras, la necesidad de comunicación, en situaciones reales, promueve una interacción significativa y constante en la lengua-meta. En cambio, el aprendizaje es un proceso consciente, resultado del conocimiento formal sobre la lengua. Por medio del aprendizaje (que depende del esfuerzo intelectual para que ocurra), el individuo es capaz de explicitar las reglas existentes en la lengua, pero eso no implica una competencia comunicativa sino lingüística. Para Krashen (1985), el aprendizaje nunca se transformará en adquisición y, por lo tanto, por medio del aprendizaje no se puede llegar a un nivel de competencia comunicativa igual al de un nativo, que ocurriría solamente si hubiera adquisición. La situación de aprendizaje contribuiría apenas con un discurso poco fluido, ya que los alumnos estarían más preocupados con la forma que con el mensaje que quieren transmitir.

Sobre la teoría de Krashen, conviene igualmente destacar la hipótesis del input. A partir de esa hipótesis, Krashen (1985) constata que las clases serían más eficientes para alumnos principiantes si los estos fuesen expuestos a ambientes de inmersión. Sin embargo, la exposición a la lengua extranjera solamente va a traer beneficios al aprendiz si el input es comprensible. Por tanto, no siempre una gran cantidad de input o mucho tiempo de exposición al input garantizan buenos resultados. Así, de acuerdo con esa teoría, lo que es más importante para la adquisición de una LE viene a ser que los alumnos tengan acceso a una cantidad de input suficiente y comprensible, una vez que el input debe de ser presentado en contexto específico. Además, se debe centrar en el mensaje, en la comunicación, y no en la estructura de la lengua.

En ese mismo sentido, Vygotsky (1998) en su abordaje socio-histórico e interaccionista, considera que las funciones psicológicas superiores se construyen en la interacción del individuo con otros individuos y con el medio, mediatizados por instrumentos y signos. Entendemos con Vygotsky (1998), mediación como un proceso de intervención de un elemento intermediario en la relación hombre/mundo. Esa mediación puede ser sistematizada en la escuela o vivenciada en la comunidad. Consideramos que la enseñanza de PLE en situación de inmersión puede ser articulada según la perspectiva vigotskiana, atendiendo a la relación intrínseca que supone aprender a partir de la tríade hombre/historia y mundo, como en el caso de los adultos que empiezan a aprender una lengua extranjera. No obstante, en el sentido de Krashen (1985), también puede ser visto como un aprendizaje que relaciona, subconscientemente, la lengua materna ya adquirida con la aprendida. 
De acuerdo con los referentes teóricos expuestos, pasamos a relatar los contextos de reflexión y análisis.

\section{Descripción del trabajo desarrollado en los dos contextos}

A modo de reflexión sobre las implicaciones de enseñar una lengua extranjera en contexto de inmersión, relacionando intrínsecamente en el aula lengua y cultura, a continuación presentamos los contextos de enseñanza y aprendizaje de PLE sobre los cuales nos propusimos investigar. El primero de ellos hace referencia a un Proyecto de Extensión de PLE realizado en una universidad en el interior del estado de São Paulo, Brasil, en situación de inmersión; y, el segundo, a un Curso de PLE que tuvo lugar en el interior de España, situación fuera de inmersión.

\section{Curso en Brasil: proyecto de PLE en situación de inmersión}

El Proyecto de Extensión llamado "Ensino de Português como Língua Estrangeira (PLE) para estrangeiros", coordinado por la Prof. Dr. Nildicéia Aparecida Rocha y por la Prof. Dr. Rosangela Silveira Sanches Gileno, promueve un programa de actividades sociales y lingüísticas dentro y fuera del aula con la finalidad de integrar al alumno extranjero en el contexto social de la UNESP y, al mismo tiempo, de la ciudad y región, así como garantizarle oportunidades de desarrollar habilidades lingüísticodiscursivas, pragmáticas, culturales e interculturales. Sin embargo, el objetivo más amplio consiste en crear espacios y momentos de convivencia y aprendizaje, en los cuales el alumno extranjero pueda adquirir la competencia en el uso del portugués como lengua extranjera (PLE) en la universidad y fuera de ella. Este contexto específico se refiere a las tres facultades de la UNESP y a un instituto. El público-meta son los alumnos intercambistas de los cursos de graduación y programas de Posgrado de las cuatro unidades, así como la comunidad externa.

En concreto, el plan de curso del proyecto en cuestión, objeto de reflexión, está estructurado a fin de contemplar las necesidades e intereses de cada grupo, por medio de diagnósticos. Llevando en consideración este contexto, la situación de inmersión y la relación indisociable entre lengua y cultura en dicho proyecto, todos los niveles de enseñanza de PLE parten siempre de una proposición cultural para inferir la sistematización lingüística, utilizando incluso una variedad representativa de los diferentes géneros textuales en lengua portuguesa, entre ellos: propaganda, música, vídeo, poesía, crónica, etc. 
Observamos que, como este proyecto se desarrolla desde 2012, en la actualidad, presenta una relativa estabilidad. Para los participantes del mismo, la situación de inmersión promueve el interés por aprender sobre los procesos culturales de Brasil, considerando que las clases parten de la perspectiva intercultural (KRAMSCH, 1993, 1998) al fomentar la negociación y el respeto hacía la lengua del otro, y al repensar sobre la propia lengua y cultura. Esta reflexión puede ser comprobada por la continuidad de los aprendices en seguir participando del proyecto durante todo el semestre o año de clases. En general, la asistencia de los alumnos ha sido de 50 alumnos por semestre. De hecho, contando a los alumnos extranjeros que las cuatro unidades de la Unesp han recibido, tiene un promedio de 60 intercambistas por semestre.

También, observamos que durante las reuniones entre las coordinadoras y los participantes del proyecto, colaboradores y becarios, es habitual comentar el destacado interés que los aprendices demuestran durante las clases acerca de aspectos históricos y culturales de Brasil y de Portugal. Por ejemplo, suscita curiosidad la formación del pueblo brasileño, la música tan diversificada, las diferencias étnicas y culturales, la forma en la que las personas tratan a los extranjeros, y otras inquietudes. Por lo tanto, se observa que al encontrarse en situación de inmersión y el deseo de pertenecer al espacio circunscrito, el brasileño, en sentido comunicativo y cultural, los aprendices cuestionan más y se interesan constantemente durante las clases por los aspectos culturales.

\section{Curso en España: fuera de inmersión}

Durante la realización de un estudio de pós-doctorado en España, como parte de las actividades, fueron impartidos cursos de PLE para la comunidad académica de la Universidad donde se realizó la investigación pós-doctoral, con el objetivo de divulgar y promover la lengua portuguesa y la cultura brasileña. En ese contexto, se ofrecieron dos cursos de PLE. El primero, llamado "Curso de Português para principiantes - Básico (A1)", proponía una primera aproximación a la Lengua Portuguesa y Cultura Brasileira, de acuerdo con el nivel A1 del Marco Europeo Común de Referencia para lenguas (QECR). Éste tuvo 26 horas de clases, distribuidas en dos encuentros semanales de 2 horas cada uno.

En general, hubo un importante interés por el curso. Así, en pocos días, había 30 inscritos: alumnos universitarios españoles de varias regiones de España e intercambistas universitarios extranjeros (varios italianos, una griega, una colombiana y una polaca). Un punto común entre los alumnos de este primero curso de portugués fue 
que la mayoría eran estudiantes de Filología Hispánica o de una Lengua Extranjera, como de Español Lengua Extranjera (ELE) o alemán. También había estudiantes del Máster en ELE y otras lenguas extranjeras, profesores de español y literatura ya formados y profesores tanto de la universidad como de la red pública de enseñanza, y estudiantes de doctorado en dicha universidad. Por lo tanto, era un grupo heterogéneo, pero con el denominador común de ser del área de Lenguas.

En cuanto al nivel lingüístico del grupo de alumnos, estos presentaban un conocimiento previo diversificado sobre el portugués, pero en general eran iniciantes. Algunos de ellos tenían un poco de conocimiento sobre la variedad hablada en Portugal y el Fado (estilo de música portuguesa), pero apenas dos alumnas (una italiana y una colombiana) tenían conocimientos del portugués hablado en Brasil, porque tenían amigos brasileños o habían estudiado portugués de Brasil mientras estudiaban en Colombia, como era el caso de la colombiana.

Metodológicamente, como dijimos anteriormente, pretendíamos trabajar los contenidos articulándolos a partir de aspectos culturales. O sea, pretendíamos presentar primero cada región brasileña, tanto en sus aspectos históricos como culturales, de los cuales desarrollaríamos las cuestiones de lengua, estructuralmente y según el nivel propuesto, A1. Sin embargo, esta propuesta no obtuvo mucha aceptación por la mayoría del grupo.

Para realizar esta práctica docente, se partió de los supuestos teóricos y metodológicos de la experiencia anterior en los cursos de PLE en Brasil (presentado anteriormente). Por lo tanto, en una situación de inmersión, construida teórica y empíricamente, considerando que: 1) enseñar una lengua extranjera ocurre mientras se adquiere/aprende y reflexiona recíprocamente sobre la cultura del(los) pueblo(s) que habla(m) esa lengua; y, específicamente, enseñar portugués para hispanohablantes presupone alguna relación posible entre esas lenguas tanto por la proximidad etimológica, debido a su origen latino, como por la respuesta positiva observada en el Proyecto de Extensión de PLE, descrito con anterioridad. No obstante, esta premisa tuvo que ser desconstruida, pues los alumnos demandaron más atención en la estructura de la lengua y no en los aspectos culturales. Tomamos el cuidado de presentar textos para iniciantes en aprendizaje de portugués como lengua extranjera, o sea, con géneros textuales indicados al nivel A1.

Con relación específicamente al tratamiento de los aspectos culturales, en general, existió una cierta resistencia por parte de los alumnos, pues el interés 
verbalizado fue aprender los aspectos lingüístico-comunicativos y no culturales. Este interés nos hizo replantear la propuesta y práctica docente, porque entendíamos teórica y metodológicamente que aprender una lengua es aprender cómo el pueblo de esa lengua se expresa comunicativa y discursivamente en su cultura, en su complejidad cultural, geográfica e histórica. Tal observación puede ser corroborada por el número de inscritos: inicialmente fueron treinta, pero apenas doce finalizaron el curso, y de estos solamente tres siguieron en el nivel 2 del curso de portugués, justamente quienes demostraron interés por la literatura y la cultura lusófonas.

Por otro lado, cuando había interés por los aspectos culturales, ése se daba más con relación a la cultura portuguesa que necesariamente brasileña, tal vez por la proximidad geográfica, histórica y económica con Portugal. Excepto las siguientes alumnas: la italiana con conocimiento en portugués variedad brasileña, la colombiana y la griega, que se mostraron encantadas por Brasil, por la lengua y cultura(s) brasileña(s).

\section{Resultados obtenidos}

De acuerdo con la pregunta inicial: ¿enseñar lengua extranjera en situación de inmersión puede traer beneficios al aprendizaje y enseñanza en la interrelación lengua y cultura? Observando los contextos presentados, según los referentes teóricos y metodológicos propuestos, podemos registrar algunas posibles interpretaciones sobre la situación de inmersión:

- El aprendizaje de PLE está propiciando el desarrollo de la competencia cultural e intercultural en los aprendices vinculados al Proyecto de Extensión, pues se observa que hay continuidad en la asistencia de esos aprendices en los talleres y actividades culturales, además de seguir en contacto vía online con los profesores de PLE que tuvieron, o sea becarios, voluntarios, colaboradores y coordinadores del proyecto (formados por alumnos de la Carrera de profesorado en Letras y profesores de esta carrera);

- La enseñanza de PLE ha corroborado los estudios sobre metodología de lenguas, porque ha sido ventajosa la enseñanza de lengua a partir de una pedagogía que afirme la relación intrínseca entre lengua y cultura. De ese modo, confirma los estudios teóricos y metodológicos del área de enseñanza y aprendizaje de lengua extranjera.

Sin embargo, en lo que se refiere al curso de no inmersión, podemos inferir por las observaciones realizadas hasta el momento, que: 
- En el aprendizaje de PLE, por no entender lingüística y comunicativamente los textos (diversos géneros textuales) que focalizan la cultura brasileña, por falta de motivación o por no existir semejanzas históricas y políticas entre Brasil y España (desde la formación de España como país) como los países de América Latina, los aprendices de PLE no demostraron mucho interés en aprender la variedad brasileña de PLE;

- En la enseñanza de PLE es necesario realizar un estudio más profundo y detallado del contexto de enseñanza y aprendizaje en el que van a ser impartidas las clases, así como un análisis de los perfiles de los aprendices, confirmando también las contribuciones teóricas sobre este tema.

Tal vez debido a la distancia geográfica e histórica entre Europa y América exista un mayor distanciamiento en el aprendizaje y, por tanto, en la enseñanza de portugués a hablantes de español de España. Sin embargo, los hispanoamericanos han demostrado un interés más significativo en el aprendizaje de PLE y, en consecuencia, la práctica docente con foco en la interrelación lengua-cultura ha sido diferente.

\section{Consideraciones finales}

Sabemos que existen muchos factores que pueden haber desencadenado el poco interés de los alumnos en curso de no-inmersión a dar continuidad, tales como la falta de motivación individual, historias de países diferentes, intereses políticos y económicos distintos, entre muchos. Estos son factores que podrían contribuir con el aprendizaje de PLE, a partir de una perspectiva cultural e intercultural. De esta forma, considerando la actitud de los alumnos de no presentar mucho interés por la cultura brasileña ni por nuestra historia, percibimos que el interés por aprender la lengua portuguesa, hablada en Brasil, todavía es muy reducido en Europa o que la perspectiva didáctica intercultural está lejos de ser una realidad en la enseñanza de lenguas de modo más amplio.

Por otro lado, con respeto a la práctica de la docente, podemos afirmar que en el curso de no inmersión, la docente tuvo que redefinir su práctica y desconstruir una “creencia" empírica sobre la importancia de enseñar lengua entrelazada a la cultura, resignificando su práctica y posibilitando el aprendizaje de PLE. Igualmente, tuvo que centrarse en la teoría para verificar que el análisis de datos sociales, históricos y culturales son norteadores y/o determinantes en el proceso de enseñanza y aprendizaje, 
específicamente sobre la enseñanza de PLE fuera de inmersión a partir de la interrelación entre lengua y cultura.

De ese modo, consideramos que la práctica reflexiva (SCHÖN, 1998) es justamente la que transforma las acciones y la que da relevancia al proceso de enseñanza y aprendizaje en su dinamicidad y heterogeneidad didáctico-pedagógica. Así, la práctica de ser docente necesita sostenerse sobre hábitos que se modifiquen y se reconstruyan a partir de la dinamicidad y de la interacción en cada contexto de enseñanza y aprendizaje auténticos.

\title{
THE PEDAGOGICAL PRACTICES OF A TEACHER OF PORTUGUESE FOREIGN LANGUAGE (PLE) IN IMMERSION AND NON-IMMERSION CONTEXT
}

\begin{abstract}
The aim of this paper is to present a reflection on the specifics of teaching Portuguese as a Foreign Language (PLE) both in the context of immersion and outside it, from the observations carried out in two stages: first, the practice of a teacher PLE will be described in immersion situation at a university in the state of São Paulo, Brazil; and then practice the same teacher in a course of PLE out of the immersion context, at a university in Spain, in a provincial capital. In this sense, the teaching practice will be analyzed from a teacher of PLE in immersion situation and beyond when the didactic and pedagogical treatment of the inseparable relationship between language and culture within an intercultural communicative approach. It is a qualitative research in which it is a case of state, showing the practice of one teacher (research subjects) in two socio-historically different contexts, but with the same approach. The survey results indicate that the teacher's practice in non-immersion context had to be re-signified to enable PLE learning in such a context. In fact, the teacher had to redefine their practice and deconstruct a belief and turn to the theory, according to their didactic and pedagogical and linguistic concerns, finding that the examination of social, historical and cultural data should always be the guiding and / or determinants as regards the teaching of a foreign language, in particular PLE out of immersion.
\end{abstract}

Key words: Teaching Practice. Portuguese Foreign Language Teaching. Immersion Context. Language And Culture.

\section{REFERÊNCIAS}

BYRAM, M. Teaching and Assessing Intercultural Communicative Competence. Clevedon: Multilingual Matters, 1997.

KRAMSCH, C. Context and Culture in Language Teaching. New York: Oxford University Press, 1993. 
Language and Culture. New York: Oxford, 1998.

KRASHEN, S. The Input Hypothesis: issues and implications. 4.ed. New York: Longman, 1985

MENDES, E. Abordagem comunicativa intercultural: uma proposta para ensinar e aprender língua no diálogo de culturas. 2004. 316 f. Tese (Doutorado em Linguística aplicada) - Universidade Estadual de Campinas, São Paulo, 2004.

Língua, cultura e formação de professores: por uma abordagem de ensino intercultural. In: MENDES, E.; CASTRO, M. L. S. (Org.). Saberes em português: ensino e formação docente. Campinas: Pontes, 2008. p.55-77.

SCHÖN, D. El profesional reflexivo: como piensam los profesionales cuando atuán. Barcelona: Paidós, 1998.

VYGOTSKY, L. S. A formação social da mente. São Paulo: Martins Fontes, 1998. 